Ciência e Natura, Santa Maria v.37 Ed. Especial-Nano e Microencapsulação de compostos bioativos e probióticos em alimentos , 2015, Dez. p. $142-151$ Revista do Centro de Ciências Naturais e Exatas - UFSM

ISSN impressa: 0100-8307 ISSN on-line: 2179-460X

\title{
Nanoestruturas contendo compostos bioativos extraídos de plantas
} Nanostructures containing bioactive compounds extracted from plants

\author{
Cristiane Franco Codevilla ${ }^{1}$, Maiara Tais Bazana ${ }^{2}$, Cristiane de Bona da Silva ${ }^{3}$, Juliano Smanioto \\ Barin ${ }^{4}$ e Cristiano Ragagnin de Menezes ${ }^{5}$
}

\begin{abstract}
1Pós-doutoranda, Departamento de Tecnologia e Ciência dos Alimentos, Universidade Federal de Santa
Maria, Santa Maria, Brasil

2Doutoranda, Departamento de Tecnologia e Ciência dos Alimentos, Universidade Federal de Santa Maria, Santa Maria, Brasil

${ }^{3}$ Doutora, Departamento de Farmácia Industrial, Universidade Federal de Santa Maria, Santa Maria, Brasil

${ }^{4}$ Doutor, Departamento de Tecnologia e Ciência dos Alimentos, Universidade Federal de Santa Maria, Santa Maria, Brasil

${ }^{5}$ Doutor, Departamento de Tecnologia e Ciência dos Alimentos, Universidade Federal de Santa Maria, Santa
\end{abstract} Maria, Brasil

\begin{abstract}
Resumo
Muitos compostos naturais, extraídos de frutas, legumes e verduras, tem potencial promissor na manutenção e promoção da saúde, bem como a prevenção e o tratamento de algumas doenças. Na indústria de alimentos, a utilização de extratos de plantas, como uma alternativa ao uso de antimicrobianos e antioxidantes químicos ou sintéticos, para combater patógenos de origem alimentar, inibir a oxidação lipídica e assim prolonga a vida de prateleira é uma tendência crescente. No entanto, a maior parte dos compostos bioativos presentes nestes extratos, como polifenóis, carotenóides, alcalóides, entre outros, apresentam baixa solubilidade em água, estabilidade e biodisponibilidade. Neste sentido, a nanotecnologia é uma abordagem inovadora que tem aplicações potenciais na investigação nutracêutica, uma vez que melhora essas características, além de melhorar a absorção de compostos bioativos, protegêe-los contra a degradação prematura no organismo e prolongar o seu tempo de circulação. Existem diferentes sistemas nanoestruturados que podem ser empregados em alimentos, como lipossomas, nanopartículas poliméricas, nanopartículas lipídicas sólidas e nanoemulsões. Este trabalho apresenta uma revisão sobre os principais sistemas nanoestruturados utilizados para associação de diferentes extratos vegetais e algumas aplicações tecnológicas.
\end{abstract}

Palavras-chave: Extratos de plantas. Nanotecnologia. Nanopartículas. Lipossomas. Nanoemulsões.

\begin{abstract}
Many natural compounds, extracted from fruits and vegetables, have promising potential for maintaining and promoting health as well as preventing and treating some diseases. In the food industry, the use of plant extracts, as an alternative to chemical or synthetic antioxidants and antimicrobials to combat the foodborne pathogens, inhibiting lipid oxidation and thus extending the shelf life is an increasing trend. However, most of bioactive compounds present in these extracts such as polyphenols, carotenoids, alkaloids, among others, have low water solubility, stability and bioavailability. In this sense, nanotechnology is an innovative approach that has potential applications in nutraceutical research, since it improves these characteristics, besides enhance their absorption, protect them from premature degradation in the body and prolong their circulation time. There are different nanostructured systems that can be used in foods such as liposome, polymeric nanoparticles, lipid solid nanoparticles and nanoemulsions. This work presents a review of the main nanostructured systems used for association of different plant extracts and some technological applications.
\end{abstract}

Keywords: Plant extracts. Nanotechnology. Nanoparticles. Liposomes. Nanoemulsions. 


\section{Introdução}

$\mathrm{A}$ limentos de origem vegetal englobam uma ampla variedade de categorias, incluindo frutas, verduras e legumes, e diversas propriedades biológicas já foram evidenciadas cientificamente, como antifúngica, antibacteriana, antioxidante, anti-inflamatória, cardioprotetora, anti-câncer, entre outras (WANG, et al., 2012, BONIFÁCIO et al., 2014). A utilização de ingredientes alimentares funcionais sintéticos gera preocupações de saúde e ecológicos. Assim, ingredientes naturais tornaram-se uma alternativa ecologicamente importante, o que tem gerado inúmeras pesquisas (Figura 1) (GORTZ et al., 2007, NUENGCHAMNONG et al., 2004, WANG, et al., 2012). A utilização de extratos de plantas ou seus produtos derivados em várias aplicações de alimentos e bebidas é uma tendência crescente na indústria de alimentos (PERUMALLA \& HETTIARACHCHY, 2011).

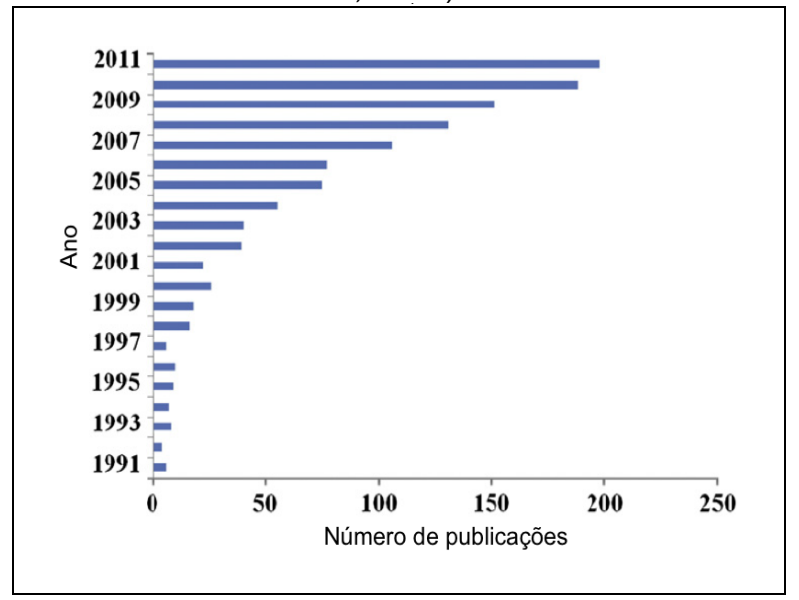

Figura 1: Distribuição das publicações relacionadas a "extratos de plantas"(1991-2011; ISI web of science).

Adaptados de WANG et al. (2012)

Os extratos de plantas são geralmente reconhecidos como seguros (do inglês generally recognized as safe, GRAS), sendo assim, permitidos para serem incorporados em sistemas alimentares. Porém, a seleção do extrato vegetal e sua aplicação depende das suas propriedades funcionais, disponibilidade, custo-eficácia, a consciência do consumidor e seus efeitos sobre os atributos sensoriais do produto final (PERUMALLA \& HETTIARACHCHY, 2011).
A eficácia de muitos extratos de plantas depende do fornecimento de compostos bioativos. Esses compostos podem ser compostos fenólicos, flavonóides, óleos essenciais, alcalóides, carotenóides, taninos, terpenóides, entre outros. No entanto, há uma limitação na aplicação em sistemas alimentares devido ao fato de, geralmente, serem pouco solúveis em água, terem baixa absorção, por não serem capazes de atravessar as membranas lipídicas das células ou terem tamanho molecular excessivamente alto, resultando em perda de biodisponibilidade e eficácia (GORTZ et al., 2007, BONIFÁCIO et al., 2014).

Soma-se a isso o fato de que a incorporação de alguns compostos bioativos em alimentos pode causar defeitos de qualidade, como gosto adstringente. Também podem atuar como substratos para reações de escurecimento, podem sofrer degradação e/ou deterioração pelas operações de processamento e armazenamento de alimentos, por exemplo, aquecimento, acidificação, luz e oxigênio (BAGHERI et al., 2013). Portanto alguns extratos acabam não sendo utilizados devido a esses obstáculos (BONIFÁCIO et al., 2014).

A tabela 1 apresenta alguns compostos bioativos, suas fontes de alimentos, potenciais benefícios para a saúde e limitações.

A fim de contornar essas limitações e utilizar extratos de plantas em sistemas alimentares, é crucial o desenvolvimento de metodologias que preservem os compostos bioativos da oxidação, degradação e reação com outros materiais durante a manipulação, processamento e armazenamento do produto final (WANG et al., 2012). Tem sido amplamente proposto utilizar a nanotecnologia, uma vez que os sistemas nanoestruturados podem ser capazes de controlar a estabilidade, melhorar a solubilidade, biodisponibilidade e controlar a liberação de compostos bioativos (ABBAS et al., 2015; NEVES et al., 2015).

Na literatura observa-se uma divisão entre os trabalhos relativos a extratos de plantas e nanotecnologia, podendo-se distinguir dois grupos bem definidos. O primeiro considera o uso de extratos de plantas para a formação de 
nanopartículas metálicas e o segundo, o uso de sistemas baseados em nanotecnologia para melhorar as propriedades biológicas e tecnológicos dos extratos de plantas (ZORZI et al., 2015). Em face do exposto, este artigo se propõem a revisar os principais sistemas nanoestruturados usados para associação de diferentes extratos de plantas, com intuito de melhorar as propriedades tecnológicas e biológicas dos extratos, trazendo benefício tanto para o alimento quanto para o consumidor.

\section{Nanotecnologia}

A nanotecnologia envolve a caracterização, fabricação e/ou manipulação de estruturas, dispositivos ou materiais que tenham pelo menos uma dimensão de cerca de 1-100 nm de

Tabela 1: Potencial benefícios para a saúde e limitações de alguns compostos bioativos

\begin{tabular}{|c|c|c|c|}
\hline $\begin{array}{l}\text { Composto } \\
\text { bioativo }\end{array}$ & Fonte & $\begin{array}{l}\text { Potencial benefício para a } \\
\text { saúde }\end{array}$ & ações \\
\hline$\beta$-caroteno & $\begin{array}{l}\text { Tomate, } \\
\text { cenoura, } \\
\text { brócolis, frutas } \\
\text { cítricas }\end{array}$ & $\begin{array}{l}\text { Antioxidante, } \\
\text { envelhecimento }\end{array}$ & $\begin{array}{l}\text { Baixa solubilidade em } \\
\text { água, } \\
\text { biodisponibilidade, pouco } \\
\text { estável quanto a oxidação }\end{array}$ \\
\hline Licopeno & Tomate & Antioxidante & $\begin{array}{l}\text { Baixa solubilidade em } \\
\text { água, } \\
\text { biodisponibilidade }\end{array}$ \\
\hline Luteína & $\begin{array}{l}\text { Espinafre, } \\
\text { banana }\end{array}$ & $\begin{array}{l}\text { Antioxidante, melhora a } \\
\text { visão }\end{array}$ & $\begin{array}{l}\text { Baixa solubilidade em } \\
\text { água, } \\
\text { biodisponibilidade }\end{array}$ \\
\hline Resveratrol & Uvas & $\begin{array}{l}\text { Diminui o HDL e aumenta o } \\
\text { LDL }\end{array}$ & $\begin{array}{l}\begin{array}{l}\text { Baixa solubilidade em } \\
\text { água, } \\
\text { biodisponibilidade }\end{array} \\
\end{array}$ \\
\hline Quercetina & $\begin{array}{l}\text { Frutas cítricas, } \\
\text { maçã, brócolis }\end{array}$ & $\begin{array}{l}\text { Diminui o colesterol, anti- } \\
\text { inflamatório }\end{array}$ & $\begin{array}{lr}\begin{array}{l}\text { Baixa solubilidade em } \\
\text { água, } \\
\text { biodisponibilidade }\end{array} & \text { baixa } \\
\end{array}$ \\
\hline
\end{tabular}

Fonte: Adaptado de Shin et al. (2015)

comprimento (DUNCAN, 2011).

A nanotecnologia é uma abordagem inovadora que tem aplicações potenciais na investigação nutracêutica. Compostos bioativos extraídos de plantas têm potencial promissor para a manutenção e promoção da saúde, bem como a prevenção e o tratamento de algumas doenças. Desse modo, as nanopartículas podem aumentar a solubilidade e estabilidade de compostos bioativos, melhorar a sua absorção, protegê-los contra a degradação prematura no organismo e prolongar o seu tempo de circulação. Além disso, as nanopartículas exibem uma elevada eficiência de absorção nas células alvo (ou tecido) através da prevenção da interação prematura com o ambiente biológico, melhoram a permeação e efeito de retenção em tecidos doentes além de melhor a sua absorção celular, resultando em toxicidade diminuída (AJAZUDDIN, 2010; BONIFÁCIO et al., 2014; WANG et al., 2014; GHORANI \& TUCKER, 2015).

Existem diferentes tipos de carreadores nanométricos, cuja preparação exige uma formulação adequada e condições de processamento oportunas. Entre eles, as nanopartículas poliméricas, nanopartículas lipídicas sólidas (NLS), os lipossomas e as nanoemulsões são adequadas para aplicações em alimentos (AJAZUDDIN, 2010; SPIGNO et al., 2013).

\subsection{Nanopartículas poliméricas}

Nanopartículas poliméricas são sistemas carreadores que podem variar entre 10-1000 nm 
de diâmetro, e dependendo da sua composição e organização estrutural, diferenciam-se em nanocápsulas e nanoesferas. As nanocápsulas contêm um núcleo oleoso cercado por uma membrana polimérica, onde o composto bioativo pode estar adsorvido à membrana polimérica e/ou dissolvido no núcleo oleoso (Figura 2). As nanoesferas, que não apresentam óleo em sua composição, são formadas por uma matriz polimérica, onde o fármaco pode ficar retido ou adsorvido (SCHAFFAZICK et al., 2003; BONIFÁCIO et al., 2014).

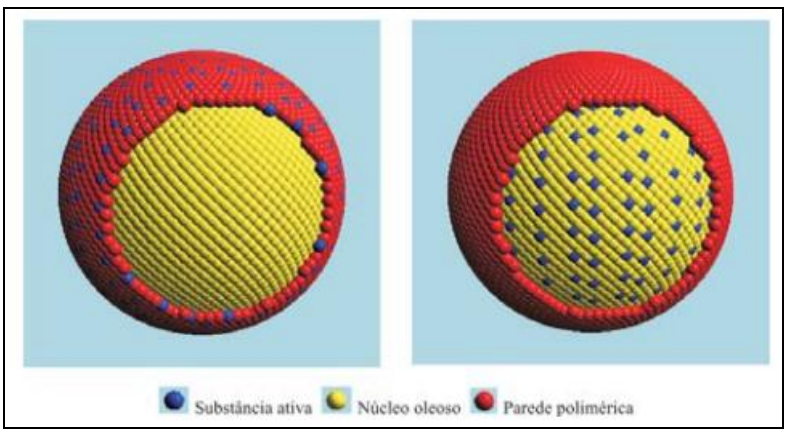

Figura 2 - Representação de nanocápsulas com o composto bioativo: (a) adsorvida à parede polimérica,

e (b) dissolvida no núcleo oleoso. Adaptado de SCHMALTZ et al. (2005)

Existem muitos métodos que podem ser utilizadas para a preparação de nanopartículas poliméricas, sendo os principais a, polimerização in situ, polimerização interfacial, deposição interfacial de polímero, nanoprecipitação, salting out, emulsificação-difusão, emulsificaçãoevaporação de solvente e coacervação complexa (MORA-HUERTAS et al., 2010). São utilizados polímeros biodegradáveis naturais ou artificiais (SCHAFFAZICK et al., 2003). Uma variedade de polímeros naturais tais como alginato de sódio, quitosana, gelatina, albumina são utilizados para desenvolver estas nanocápsulas não-tóxicas, biodegradáveis e de baixo custo (KUMAR et al., 2015). Independentemente do método escolhido, os produtos são obtidos na forma de suspensões coloidais aquosas (BONIFÁCIO et al., 2014).

Pelo fato da indústria de processamento de frutas gerar uma grande percentagem de subprodutos produzidos nas diferentes fases do tratamento, como cascas e sementes, que não são utilizadas e que muitas vezes apresentam elevados teores de compostos bioativos, Silva e colaboradores (2014) desenvolveram nanopartículas empregando extratos dos subprodutos das frutas acerola, maracujá e goiaba.
A fraca solubilidade em água e baixa biodisponibilidade justificaram a nanoencapsulação. Todos os sub-produtos foram nanoencapsulados e apresentaram atividade antimicrobiana contra Listeria monocytogenes Scott A e Escherichia coli K12.

Óleos, gorduras e alimentos que contenham lípidios insaturados podem ser afetados pela oxidação lipídica, com redução da vida de prateleira, palatabilidade, funcionalidade e qualidade nutricional (NORONHA et al., 2013; MOHAMMADI et al., 2016). Os antioxidantes podem ser adicionados em formas naturais ou sintéticos em alimentos, quer para retardar o início da oxidação ou para diminuir a taxa de oxidação lipídica. Devido ao seus potenciais efeitos tóxicos, antioxidantes sintéticos têm sido em grande parte substituídos por antioxidantes naturais (NUENGCHAMNONG et al., 2004; NORONHA et al., 2013, QUINTÃO et al., 2013). Como uma possível alternativa, Noronha et al. (2013) desenvolveram nanocápsulas contendo $\alpha$ tocoferol, com eficiência de encapsulação de 99,97\%, tamanho de partícula de 184,6 nm e índice de polidispersão de 0,112.

A quercetina é um dos flavonóides mais encontrados nas plantas e possui comprovada atividade antioxidante. Kumari et al. (2010) nanoencapsularam quercetina com o intuito de melhorar sua fraca solubilidade aquosa e estabilidade. $\mathrm{O}$ tamanho médio das nanopartículas foi de $130 \mathrm{~nm}$ e a eficiência de encapsulação de $96,7 \%$. Os antioxidantes naturais podem ser usados como uma alternativa na substituição de aditivos alimentares sintéticos, tais como hidroxitolueno butilado (BHT) hidroxianisol butilado ou (BHA) (NUENGCHAMNONG et al., 2004).

\subsection{Lipossomas}

São estruturas vesiculares (Figura 3) compostas por moléculas anfifílicas, tais como os fosfolipídios, possuindo elevada biocompatibilidade e biodegradabilidade (DIMER et al., 2013). Seu tamanho pode variar de nanômetros até vários micrômetros, sendo tanto a técnica quanto os componentes importantes para definir suas propriedades físico-químicas finais, como carga de superfície, tamanho e estabilidade. 


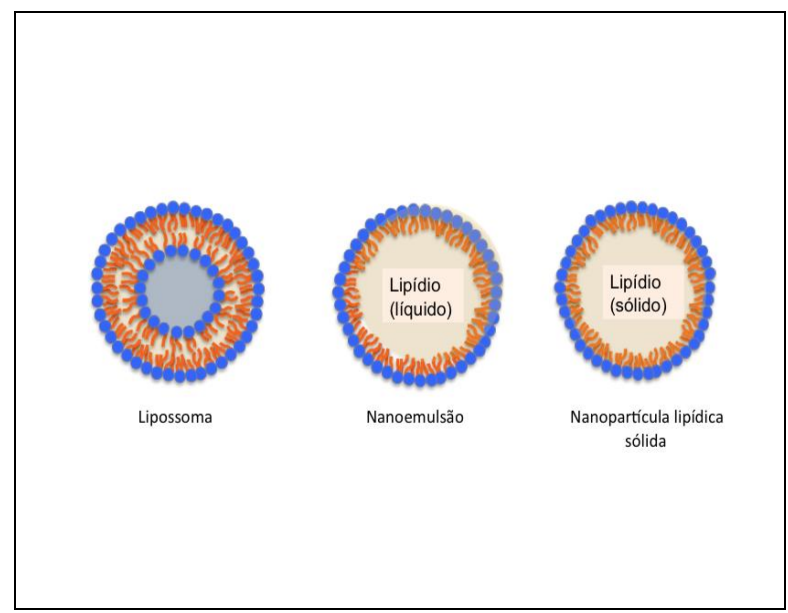

Figura 3. Representação de lipossomas, nanoemulsões e nanopartículas lipídicas sólidas. Adaptado de Wang et al. (2014)

Os lipossomas são constituídos por uma ou mais bicamadas lipídicas concêntricas em torno do compartimento aquoso interno e são classificados de acordo com o número de camadas e tamanho. Lipossomas com uma bicamada lipídica são chamados unilamelares e com várias bicamadas lipídicas de multilamelares. Quanto ao tamanho, as vesículas unilamelares podem ser pequenas $(<30 \mathrm{~nm})$ ou grandes (30-100 nm), sendo caracterizadas como lipossomas unilamelares pequenos - SUV (small unilamellar vesicles) e lipossomas unilamelares grandes - LUV (large unilamellar vesicles). (AJAZUDDIN, et al., 2010; ANANDHARAMAKRISHNAN, 2014).

Podem encapsular tanto compostos hidrofílicos quanto lipofílicos. Os compostos bioativos hidrossolúveis ficam encapsulados no interior da cavidade enquanto os lipofílicos são inseridos ou adsorvidos na bicamada lipídica. Alternativamente, ambos os tipos de substância podem ser encapsuladas (BATISTA et al., 2007;; BONIFÁCIO et al., 2014).

As tecnologias mais comuns para a preparação de lipossomas são hidratação do filme, evaporação em fase reversa, método da injeção do etanol, entre outras. A escolha do método depende do tipo e tamanho do lipossoma que se pretende produzir e a aplicação (BATISTA et al., 2007). As aplicações dos lipossomas em alimentos incluem encapsulação de nutrientes, nutracêuticos, aditivos alimentares, e antimicrobianos (LU et al., 2011).

Polifenóis do chá verde são muitas vezes utilizados como antioxidantes, porém são instáveis frente ao oxigênio e luz, reduzindo a sua estabilidade no processamento e armazenamento, o que limita sua aplicação. Lu et al. (2011) desenvolveram lipossomas com polifenóis do chá e obtiveram um produto estável por longo período de tempo, quando armazenado no congelador, melhorando sua estabilidade.

Extrato polifenólico de semente de uva foi encapsulado em lipossomas por homogeneização a alta pressão (GIBIS et al., 2012). O diâmetro médio dos lipossomas contendo extrato de uva foi inferior a $100 \mathrm{~nm}$ e apresentaram estabilidade por um longo período.

Gibis et al. (2014) desenvolveram lipossomas com extrato de hibisco, devido sua elevada quantidade de compostos polifenóis, como antocianinas, e também realizaram deposição de duas camadas adicionais. Observaram que a incorporação de extrato de hibisco como um antioxidante conduziu à maior estabilidade oxidativa da lecitina de soja e a quantidade de extrato de hibisco incorporados em lipossomas mostrou um elevado teor de antocianinas no núcleo interno, estando fracamente adsorvido na superfície dos lipossomas.

Murta-comum é uma planta da família Myriaceae. Gortzi et al. (2008), testaram as atividades antimicrobiana e antioxidante de lipossomas contendo extrato de murta-comum e compararam com o extrato não encapsulado, antioxidantes sintéticos BHT e $\alpha$-tocoferol, e antimicrobianos netilmicina e intraconazol. Todas as concentrações testadas apresentaram capacidade antioxidante, porém abaixo do BHT e no concentração de 160 ppm acima do $\alpha$ tocoferol. Os lipossomas apresentaram capacidade antioxidante 25 vezes maior que o extrato livre. A atividade antimocrobiana dos lipossomas também foi superior ao do extrato livre, principalmente contra bactérias Gram positivas. Concluíram então que os lipossomas contendo murta-comum podem ser uma alternativa como antioxidante e conservante natural em alimentos.

\subsection{Nanopartículas lipídicas sólidas}

Nanopartículas lipídicas sólidas (NLS) (Figura 3), desenvolvidas no início dos anos 1990, são formadas por uma dispersão de 
lipídeos com alto ponto de fusão. São derivadas de emulsões O/A por substituição do óleo por um lipídio sólido, o qual permanece nesse estado na temperatura corporal. Esses sistemas, com diâmetro médio na faixa de aproximadamente 50 a $1000 \mathrm{~nm}$, representam uma alternativa a lipossomas e nanopartículas poliméricas (PARDEIKE et al., 2009; BONIFÁCIO et al., 2014). Estudos relatam que uma das vantagens das NLS é o não requerimento de uso de solventes orgânicos na sua produção. Essas nanopartículas demonstram grande capacidade de encapsular compostos hidrofílicos e lipofílicos em sua matriz lipídica (PARDEIKE et al., 2009; DIMER et al., 2013).

Em comparação com nanoemulsões e lipossomas, NLS tem algumas vantagens distintas, que incluem: alta eficiência de encapsulamento, não utilizam solventes orgânicos; possibilidade de produção em larga escala e esterilização; flexibilidade em controlar a liberação dos compostos encapsulados devido à matriz sólida; taxa de degradação mais lenta e de liberação prolongada do composto bioativo, proteção eficaz dos compostos bioativos contra a degradação química (MEHNERT \& MADER, 2001; PATIDAR et al., 2010; ANANDHARAMAKRISHNAN, 2014).

A preparação destes sistemas pode ser realizada por diferentes técnicas, incluindo homogeneização de alta pressão, emulsificaçãosonicação, microemulsão e técnicas de emulsãoevaporação do solvente. Porém, a homogeneização à alta pressão tem sido a mais utilizada devido ao seu fácil escalonamento para aplicação industrial e curto tempo de processo (DIMER et al., 2013, ANANDHARAMAKRISHNAN, 2014; BONIFÁCIO et al., 2014).

De maneira geral, o homogeneizador de alta pressão pode ser alimentado com a dispersão de lipídios e o composto bioativo a quente ou a frio. No processo a quente, a pré-emulsão é passada no homogeneizador ainda quente para a obtenção de nanoemulsões. Após, a nanoemulsão homogeneizada é resfriada a temperatura ambiente dando origem a NLS. No processo a frio a dispersão do composto bioativo no lipídio é inicialmente solidificada em nitrogênio líquido, moída, o pó disperso diluído com o auxílio de um tensoativo e posteriormente submetido à homogeneização (PARDEIKE et al., 2009, ANANDHARAMAKRISHNAN, 2014).

Segundo Salminen et al. (2016), a fortificação de alimentos com $\beta$-caroteno, vitamina-A e ácidos graxos poli-insaturados tem sido de elevado interesse devido à crescente conscientização nutricional, bem como a prevenção da desnutrição em países em desenvolvimento. As principais dificuldades para a fortificação de alimentos com lípidios funcionais são a sua elevada susceptibilidade para oxidação e sua baixa solubilidade em alimentos aquosos. SALMINEN et al. (2016) obtiveram NLS contendo $\beta$-caroteno, NLS contendo vitamina-A e NLS contendo ômega-3, e concluíram que os principais fatores que influenciam o arranjo estrutural e a estabilidade química dos lípidios funcionais em dispersões de lípidios sólidas são a sua solubilidade na fase aquosa e a sua temperatura de cristalização em relação à do transportador lipídico.

$\mathrm{O}$ consumo de alimentos funcionais pode trazer uma série de benefícios à saúde, no entanto, a maioria dos compostos apresenta baixa biodisponibilidade oral (XIE et al., 2011). Exemplo disso é a curcumina, composto fenólico extraído do açafrão, com característica hidrofílica e baixa biodisponibilidade oral, mas que oferece uma série de benefícios potenciais no tratamento de várias doenças crônicas, como neurodegenerativa, cardiovasculares, pulmonares e auto-imunes (KAKKAR et al., 2011; XIE et al., 2011). Kakkar et al. (2011) associaram curcumina com NLS, para melhorar sua biodisponibilidade oral. Obtiveram NLS com tamanho médio de partícula de $134 \mathrm{~nm}$, estável por 12 meses a $5 \pm 3^{\circ} \mathrm{C}$ e com alta biodisponibilidade.

Calêndula é uma planta medicinal onde estão presentes carotenóides e seu extrato tem demonstrado atividade anti-inflamatória e cicatrizante associados a triterpenos, principalmente monoésteres de faradiol. Esses compostos bioativos não são solúveis em água e solventes orgânicos são necessários para a sua entrega à camada aquosa e a superfície ocular. Portanto, Arana et al. (2015), nanoencapsularam extratos de calêndula e obtiveram um produto seguro, livre de solventes, com boa estabilidade, sendo útil para condições de armazenamento prolongados. 


\subsection{Nanoemulsões}

Nanoemulsões (Figura 3) são dispersões de dois líquidos imiscíveis estabilizados por um tensoativo ou uma mistura de tensoativos, com diâmetro médio de gota em tamanho nanométrico (20 a $500 \mathrm{~nm})$ e transparência dependente do tamanho da partícula (translúcida $<\approx 200 \mathrm{~nm}>$ leitosa) (QUINTÃO et al., 2013; ZORZI et al., 2015). Quanto menor o diâmetro de gotículas maior estabilidade frente à cremação e sedimentação (SOLANS et al., 2005).

O uso de tensoativos é o fator responsável pela estabilidade das nanoemulsões e esses compostos anfifílicos posicionam-se entre as duas fases da emulsão (aquosa e oleosa), originando uma película interfacial que estabiliza o sistema (FERNANDEZ et al., 2004). As nanoemulsões podem ser óleo em água (O/A) ou água em óleo $(\mathrm{A} / \mathrm{O})$, dependendo da hidrofilia ou lipofilia do meio dispersante. Nanoemulsões de sucesso não são apenas fisicamente estáveis, sem desnatar, sem agregação de gotículas e mudanças na dimensão das gotículas, mas também eficazes na proteção de compostos bioativos lipófilos contra os processos de degradação (GUAN et al., 2016).

As nanoemulsões podem ser preparadas através de métodos de alta e de baixa energia. No primeiro caso estão a homogeneização à alta pressão, sonicação e microfluidização, os quais requerem equipamentos específicos. No segundo caso, métodos que utilizam a energia química armazenada nos componentes como, por exemplo, a emulsificação espontânea e o método da temperatura de inversão de fases (SOLANS et al., 2005; ANTON et al., 2008; ANANDHARAMAKRISHNAN, 2014). Para associação de extratos de plantas em nanoemulsões, os métodos mais empregados são a emulsificação espontânea e a homogeneização a alta pressão (ZORZI et al., 2015).

SPIGNO et al. (2013) preparam nanoemulsões contendo extrato fenólico de bagaço de uva, a fim de aumentar a sua solubilidade lipídica e eficiência antioxidante. Posteriormente, aplicaram como um agente de conservação natural em pasta de avelã. $\mathrm{O}$ estudo mostrou a eficiência do extrato fenólico de bagaço de uva em melhorar o período de vida útil de avelã por inibir a sua oxidação. A nanoemulsão A/O demonstrou ser a melhor solução de encapsulamento como potencial agente conservante natural, enquanto na emulsão O/A houve a liberação mais lenta de compostos fenólicos, podendo ser potencialmente explorado para a produção de um ingrediente funcional saudável.

O consumo de carotenóides podem reduzir a incidência de certas doenças crônicas, mas a sua utilização em alimentos está atualmente limitada devido à sua fraca solubilidade em água, baixa biodisponibilidade e instabilidade química (QIAN et al., 2012). Entre os carotenóides, $\beta$ caroteno tem a maior atividade pró-vitamina $\mathrm{A}$, sendo um forte candidato à incorporação em alimentos funcionais. No entanto, a absorção de $\beta$-caroteno a partir de alimentos naturais e processados é muitas vezes ineficiente e altamente variável (QIAN et al., 2012; BONIFÁCIO et al., 2014). Nesse sentido, Qian et al. (2012) avaliaram a biodisponibilidade do $\beta$ caroteno quando associado a nanoemulsões. Obtiveram uma nanoemulsão O/A, com diâmetro médio de gota de 200 nm, estável frente à passagem pelos fluidos gastrointestinais simulados.

Óleos essenciais de plantas, especialmente tomilho, orégano e cravo, tem mostrado forte efeito antimicrobiano, porém a sua aplicação na proteção de alimentos é ainda limitada devido à sua baixa solubilidade em água (BHARGAVA et al., 2015). Bhargava et al. (2015) desenvolveram nanoemulsões de óleo de orégano e avaliaram quanto à sua eficácia na inativação do crescimento de bactérias de origem alimentar na alface fresca. As nanoemulsões de óleo de orégano foram capazes de reduzir a Listeria monocytogenes, S. Typhimurium, e E. coli O157: H7 em até 3,57, 3,26, e 3,35 log UFC/g, respectivamente, na alface fresca, sugerindo que nanoemulsões com óleos essenciais podem ser alternativas naturais aos produtos químicos no controle de segurança de produtos.

Silva Júnior et al. (2013) desenvolveram nanoemulsões contendo óleo de semente de romã. A romã é uma fruta com mais substâncias antioxidantes do que o suco de uva e o vinho, possuindo também compostos anti-inflamatórios e anti-infecciosos. Obtiveram nanoemulsões estáveis contendo $1,5 \% \mathrm{~m} / \mathrm{m}$ de óleo de semente de romã e $5 \% \mathrm{~m} / \mathrm{m}$ de uma mistura de tensoativos. $\mathrm{O}$ tamanho médio obtido para as 
gotículas foi de $211 \mathrm{~nm}$ e as nanoemulsões foram estáveis por um período de 90 dias.

\section{Conclusões}

A nanotecnologia é uma das tecnologias mais inovadoras deste século e a sua utilização no setor de alimentos é crescente. Existem diversos sistemas nanoestruturados que podem ser aplicados, cada um com suas particularidades. As pesquisas demonstram os benefícios de associar compostos bioativos extraídos de plantas (frutas, legumes e verduras) a sistemas nanoestruturados, tanto prevenindo ou promovendo a saúde do consumidor, quanto trazendo benefícios para o produto, melhorando sua qualidade e segurança.

\section{Referências}

Abbas, S., Karangwa, E., Bashari, M., Hayat, K., Hong, X., Sharif, H. R., Zhang, X. (2015) Fabrication of polymeric nanocapsules from curcumin-loaded nanoemulsion templates by self-assembly. Ultrasonics Sonochemistry 23, 8192.

Anandharamakrishnan, C. (2014). Techniques for Nanoencapsulation of Food Ingredients, Springer.

Anton, N., Benoit, J. P., Saulnier, P. (2008). Design and production of nanoparticles formulated from nano-emulsion templates-A review. Journal of Controlled Release 128, 185199.

Arana, L., Salado, C., Vega, S., AizpuruaOlaizola, O., Arada, I., Suarez, T., Usobiaga, A., Arrondo, J. L. R., Alonso, A., Goni, F. G., Alkorta, I. (2015). Solid lipid nanoparticles for delivery of Calendula officinalis extract. Colloids and Surfaces B: Biointerfaces, 135, 18-26.

Ajazuddin, S. S. (2010). Applications of novel drug delivery system for herbal formulations. Fitoterapia, 81, 680-689.
Batista, C. M., Carvalho, C. M. B., Magalhães, N. S. (2007). Lipossomas e suas aplicações terapêuticas: Estado da arte. Revista Brasileira de Ciências Farmacêuticas, 43(2), 167-179.

Bhargava, K., Conti, D. S., Rocha, S. R. P., Zhang, Y. (2015). Application of an oregano oil nanoemulsion to the control of foodborne bacteria on fresh lettuce. Food Microbiology, 47, 69-73.

Bonifácio, B. V., Silva, P. B., Ramos, M. A. S., Negri, K. M. S., Bauab, T. M., Chorilli, M. (2014). International Journal of Nanomedicine, 9, 1-15.

Dimer, F. A., Friedrich, R. B., Beck, R. C. R., Guterres, S. S., Pohlmann, A. R. (2013). Impactos da Nanotecnologia Na Saúde: Produção De Medicamentos. Quimica Nova, 36(10), 1520-1526.

Duncan, T. V. Applications of nanotechnology in food packaging and food safety: Barrier materials, antimicrobials and sensors. Journal of Colloid and Interface Science 363 (2011) 1-24.

Fernandez, P., Andre, V., Rieger, J., Kuhnle, K. (2004). Nano-emulsion formation by emulsion phase inversion. Colloids and Surfaces A: Physicochem. Eng. Aspects, 251, 53-58.

Ghorani, B., Tucker, N. (2015). Fundamentals of electrospinning as a novel delivery vehicle for bioactive compounds in food nanotechnology. Food Hydrocolloids, 51, 227-240.

Gibis, M., Vogt, E., Weiss, J. (2012). Encapsulation of polyphenolic grape seed extract in polymer-coated liposomes. Food \& Function, 3, 246-254.

Gibis, M., Zeeb, B., Weiss, J. (2014). Formation, characterization, and stability of encapsulated hibiscus extract in multilayered liposomes. Food Hydrocolloids, 38, 28-39. 
Gortzi, O., Lalas, S., Chinou, I., Tsaknis, J. (2007). Evaluation of the Antimicrobial and Antioxidant Activities of Origanum dictamnus Extracts before and after Encapsulation in Liposomes. Molecules, 12, 932-945.

Gortzi, O., Lalas, S., Chinou, J., Tsaknis, J. (2008). Reevaluation of bioactivity and antioxidant activity of Myrtus communis extract before and after encapsulation in liposomes. European Food Research and Technology, 226, 583-590.

Guan, Y., Wu, J., Zhong, O. (2016). Eugenol improves physical and chemical stabilities of nanoemulsions loaded with $\beta$-carotene. Food Chemistry, 194, 787-796.

Kakkar, V., Singh, S., Singla, D., Kaur, I. P. (2011). Exploring solid lipid nanoparticles to enhance the oral bioavailability of curcumin. Molecular nutrition \& food research, 55, 495-503.

Kumar, S., Chauhan, N., Gopal, M., Kumar, R. Dilbaghi, N. (2015). Development and evaluation of alginate-chitosan nanocapsules for controlled release of acetamiprid. International Journal of Biological Macromolecules, 81, 631-637.

Kumari, A., Yadav, S. K., Pakade, Y. B., Singh, B., Yadav, S. C. (2010). Development of biodegradable nanoparticles for delivery of quercetin. Colloids and Surfaces B: Biointerfaces, 80, 184-192.

Lu, Q., Li, D. C., Jiang, J. G. (2011). Preparation of a Tea Polyphenol Nanoliposome System and Its Physicochemical Properties. Journal of Agricultural and Food Chemistry, 59, 1300413011.

Mehnert, W., Mader, K. (2001). Solid lipid nanoparticles Production, characterization and applications. Advanced Drug Delivery Reviews, 47, 165-196.
Mohammadi, A., Jafari, S. M., Esfanjani, A. F., Akhavan, S. (2016). Application of nanoencapsulated olive leaf extract in controlling the oxidative stability of soybean oil Food Chemistry, 190, 513-519.

Mora-Huertas, C. E.; Fessi, H., Elaissari, A. (2013). Polymer-based nanocapsules for drug delivery. International Journal of Pharmaceutics, 385, 113-142.

Neves, M. A., Hashemi, J., Prentice, C. (2015). Development of novel bioactives delivery systems by micro/nanotechnology. Current Opinion in Food Science, 1, 7-12.

Noronha, C. M., Granada, A. F., Carvalho, S. M., Lino, R. C., Maciel, M. V. O. B., Barreto, P. L. M. (2013). Optimization of $\alpha$-tocopherol loaded nanocapsules by the nanoprecipitation method. Industrial Crops and Products, 50, 896-903.

Nuengchamnong, N., Hermans-Lokkerbol, A., Ingkaninan, K. (2004). Separation and Detection of the Antioxidant Flavonoids, Rutin and Quercetin, Using HPLC Coupled on-line With Colorimetric Detection of Antioxidant Activity. Naresuan University Journal, 12(2): 25-37.

Pardeike, J., Hommoss, A., Muiller, R. H. (2009). Lipid nanoparticles (SLN, NLC) in cosmetic and pharmaceutical dermal products. International Journal of Pharmaceutics, 366, 170-184.

Patidar, A., Thakur, D. S., Kumar, P., Verma, J. (2010). A Review on Novel Lipid Based Nanocarriers. International Journal of Pharmacy and Pharmaceutical Sciences, 2(4), 30-35.

Perumalla, A. V. S., Hettiarachchy, N. S. (2011). Green tea and grape seed extracts - Potential applications in food safety and quality. Food Research International, 44, 827-839.

Qian, C., Decker, E. A., Xiao, H., McClements, D. J. (2012). Nanoemulsion delivery systems: 
Influence of carrier oil on $\beta$-carotene bioaccessibility. Food Chemistry, 135, 1440-1447.

Quintão, F. J. O., Tavares, R. S. N., Vieira-Filho, S. A., Souza, G. H. B., Santos, O. D. H. (2013). Hydroalcoholic extracts of Vellozia squamata: study of its nanoemulsions for pharmaceutical or cosmetic applications. Revista Brasileira de Farmacognosia, 23(1), 101-107.

Salminen, H., Gömmel, C., Leuenberger, B. H., Weiss, J. (2016). Influence of encapsulated functional lipids on crystal structure and chemical stability in solid lipid nanoparticles: Towards bioactive-based design of delivery systems. Food Chemistry, 190, 928-937.

Schaffazick, S. R., Guterres, S. S., Freitas, L. L., Pohlmann, A. R. (2003). Caracterização e estabilidade físico-química de sistemas poliméricos nanoparticulados para administração de fármacos. Quimica Nova, 26(5), 726-737, 2003.

Schmaltz, C., Santos, J. V., Guterres, S. S. (2005). Nanocápsulas como uma tendência promissora na área cosmética: A imensa potencialidade deste pequeno grande recurso. Infarma, 16, 1314.

Shin, G. H., Kim, J. T., Park, H. J. (2015). Recent developments in nanoformulations of lipophilic functional foods. Trends in Food Science \& Technology, in Press.

Silva, L. M., Hill, L. E., Figueiredo, E., Gomes, C. L. (2014). Delivery of phytochemicals of tropical fruit by-products using poly(DL-lactide-coglycolide) (PLGA) nanoparticles: Synthesis, characterization, and antimicrobial activity. Food Chemistry, 165, 362-370.

Silva Junior, E., Zanon Junior, G. B., Zanella, I., Raffin, R., Cielo, V., Rossato, J., Bulhões, L. O. S. (2013). Formação de nanoemulsões do tipo óleo em água contendo óleo de semente de romã.
Disciplinarum Scientia. Série: Ciências Naturais e Tecnológicas, 14(1), 115-122.

Spigno, G., Donsì, F., Amendola, D., Sessa, M, Ferrari, G., D. Faveri, M. (2013). Nanoencapsulation systems to improve solubility and antioxidant efficiency of a grape marc extract into hazelnut paste. Journal of Food Engineering, 114, 207-214.

Solans, C., Izquierdo, P., Nolla, J., Azemar, N. (2005). Nano-emulsions. Current Opinion in Colloid \& Interface Science, 10(3-4), 102-110.

Wang, S., Marcone, M F., Barbut, S., Lim, L. T. (2012). Fortification of dietary biopolymersbased packaging material with bioactive plant extracts. Food Research International, 49, 80-91.

Wang, S., Su, R., Nie, S., Sun, M., Zhang, J., Wu, D., Moustaid-Moussa, N. (2014). Application of nanotechnology in improving bioavailability and bioactivity of diet-derived phytochemicals. Journal of Nutritional Biochemistry, 25, 363-376.

Xie, X., Tao, Q., Zou, Y., Zhang, F., Guo, M., Wang,Y., Wang, H., Zhou, Q., Yu, S. (2011). PLGA Nanoparticles Improve the Oral Bioavailability of Curcumin in Rats: Characterizations and Mechanisms. Journal of Agricultural and Food Chemistry, 59, 9280-9289.

Zorzi, G. K., Carvalho, E. L. S., Poser, G. L., Teixeira, H. F. (2015). On the use of nanotechnology-based strategies for association ofcomplexes matrices from plant extracts. Revista Brasileira de Farmacognosia, in press. 